\title{
DOENÇAS INFECTOCONTAGIOSAS E A PESSOA IDOSA: PERSPECTIVAS PARA O ENSINO DA ENFERMAGEM PÓS-PANDEMIA COVID-19
}

Carlos Jordão de Assis Silva' ORCID: 0000-0002-9575-9030

Bruna Ruselly Dantas Silveira' ORCID: /0000-0002-8854-148X

Valéria Gomes Fernandes da Silva' ORCID: 0000-0003-1381-8664

Meine Siomara Alcântara"

ORCID: 0000-0003-4977-3189

Rejane Maria Paiva de Menezes' ORCID: 0000-0002-0600-0621

Luciane Paula Batista Araújo de Oliveira' ORCID: 0000-0003-1629-8991

'Universidade Federal do Rio Grande do Norte. Natal, Rio Grande do Norte, Brasil.

"Secretaria Municipal de Saúde. Natal, Rio Grande do Norte, Brasil.

Autor Correspondente: Luciane Paula Batista Araújo de Oliveira E-mail: lucianepoliveira@yahoo.com.br

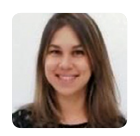

Como citar: Silva CJA, Silveira BRD, Silva VGF, Alcântara MS, Menezes RMP, Oliveira LPBA. Doenças infectocontagiosas e a pessoa idosa: perspectivas para o ensino da enfermagem pós-pandemia COVID-19. In: Santana RF (Org.). Enfermagem gerontológica no cuidado do idoso em tempos da COVID 19. Brasilia, DF: Editora ABen; 2021. 171 p. (Serie Enfermagem e Pandemias, 5). https://doi.org/10.51234/aben.21.e05.c20

\section{INTRODUÇÃO}

No final do ano de 2019 foi descoberto na China o novo coronavírus (SARS-CoV-2) responsável por causar a COVID-19. A contaminação acontece por meio da transmissão de aerossóis liberados através do espirro, tosse e secreções respiratórias, podendo causar um quadro clínico que varia de assintomático a síndrome respiratória grave. Seu alcance tomou proporções significativas, disseminando-se rapidamente e contaminando diversas pessoas ao redor do mundo, deflagrando-se uma pandemia, anunciada pela Organização Mundial da Saúde (OMS) no dia 11 de março de $2020^{(1-2)}$

De acordo com dados do Ministério da Saúde (2020), até o dia 25 de julho de 2020 foram confirmados mais de 15 milhões de casos e 639.275 mortes pela COVID-19 no mundo. Neste mesmo período, o Brasil atingiu a marca de 2.394.513 casos confirmados e 86.449 óbitos, dentre os quais 83.553 foram decorrentes da Síndrome Respiratória Aguda Grave por COVID-19 e desse quantitativo, 72,1\% tinha 60 anos ou mais e $61,5 \%$ apresentavam pelo menos uma comorbidade associada ${ }^{(3-4)}$.

Este cenário pandêmico aflorou a necessidade de discussão permanente sobre as doenças infectocontagiosas enquanto problema de saúde pública que atinge toda a população, em especial os idosos, tendo em vista que os dados apontam para um alto risco de mortalidade desse grupo etário pela COVID-19. Tal fato se deve a fatores como a imunossenescência resultante das alterações fisiológicas do processo de envelhecimento, além da prevalência de doenças crônicas nessa faixa etária aumentando as chances de desenvolver complicações, caso seja acometido pela COVID-19(5-6).

Assim, como reflexo desta pandemia, urge a necessidade de uma prática gerontológica planejada para o as necessidades de doenças emergentes e reemergentes, bem como, profissionais de saúde com competência 
técnico-científica de cuidar das pessoas idosas, visto que o processo do envelhecer é um fenômeno natural, diversificado, permeado por mudanças de ordem biológica, fisiológica e anatômica e psicossocial, e como tal, que demanda por cuidado holístico e integral ${ }^{(6-7)}$.

A enfermagem como categoria profissional com o maior contingente de profissionais da equipe multiprofissional de saúde, cujo cerne de sua assistência é o cuidado, deve estar qualificada para assistir os pacientes idosos nos diferentes níveis de atenção à saúde, atendendo as demandas de saúde individuais e coletivas desse público, sendo o risco às doenças infectocontagiosas uma demanda que se destacou com a chegada do novo coronavírus no mundo(7-8).

Para isso, é imprescindível que, na formação de enfermeiros e técnicos de enfermagem sejam abordados conteúdos voltados especificamente à saúde da pessoa idosa, agregando possíveis novas tendências pós-pandemia, destacando-se a atenção às doenças infectocontagiosas, haja vista o risco que esse grupo etário tem de contrair tais afecções ${ }^{(8)}$.

Frente a essas considerações, a realização do presente estudo se justifica pela importância de uma reflexão sobre as doenças infectocontagiosas no âmbito do ensino de enfermagem em gerontologia no período pós-pandemia, face à vulnerabilidade dessa população a doenças dessa natureza.

\section{OBJETIVO}

Refletir acerca do ensino sobre doenças infectocontagiosas e a saúde da pessoa idosa na formação do enfermeiro pós-pandemia COVID-19.

\section{MÉTODOS}

Trata-se de um estudo descritivo, qualitativo, de natureza teórica-reflexiva, que versa sobre apontamentos importantes relacionados às perspectivas do ensino de enfermagem pós-pandemia COVID-19 no contexto das doenças infectocontagiosas na pessoa idosa. Para fundamentar esta reflexão, foram realizadas buscas na literatura nacional e internacional de estudos e documentos relacionados, que subsidiaram as leituras e a análise crítica. Essas foram organizadas em três categorias analíticas: Necessidade do ensino de enfermagem gerontológica; A pessoa idosa e às doenças infectocontagiosas; e Perspectivas para o ensino de enfermagem gerontológica pós-pandemia.

\section{RESULTADOS}

\section{Necessidade do ensino de enfermagem gerontológica}

O processo de envelhecimento da atual sociedade é um fato marcante. No mundo, o número de pessoas idosas com 60 ou mais em 1950 era de 202 milhões, em 2020 1,1 bilhão, e em 2100 a estimativa é de 3,1 biIhões, ou seja, um crescimento absoluto de 15,2 vezes. No Brasil, o envelhecimento tem sido mais acelerado que o cenário global. No mesmo período, o crescimento absoluto foi de 27,6 vezes, sendo que em 2020 o número de pessoas idosas passou para 29,9 milhões, com estimativa de alcançar os 72,4 milhões em 2100, mostrando que o futuro será grisalho, tanto no Brasil como no mundo(9)

A longevidade em nosso país é uma realidade, e apresenta características peculiares com grandes desigualdades sociais no processo de envelhecimento. Soma-se a isso mudanças ocorridas na composição da família brasileira, com a queda da fertilidade, incremento do papel da mulher no mercado de trabalho, caracterizando grandes desafios a serem enfrentados nas políticas de saúde, assistência social e previdência social ${ }^{(10)}$ Assim, percebe-se que as nações terão que se preparar do ponto de vista econômico, social, previdenciário e de saúde para o novo perfil populacional. 
Diante da problemática descrita, o ensino de enfermagem gerontológica caracteriza-se como uma necessidade urgente, tendo em vista os desdobramentos da transição demográfica culminando em um aumento da população idosa e, consequentemente, maior demanda de idosos nos serviços de saúde.

Urge então a necessidade de conhecimento teórico e prático para o cuidado de enfermagem em gerontologia, uma vez que a pessoa idosa apresenta mecanismos fisiológicos específicos do processo de envelhecimento associados, em muitos casos, à presença de comorbidades e patologias caracterizando o processo de senilidade ${ }^{(11)}$

Embora previsto nas Diretrizes Curriculares Nacionais (DCNs) dos cursos de graduação em Enfermagem que os egressos devem possuir habilidades e competências para atuar na assistência à saúde dos diferentes processos de vida, incluindo na saúde da pessoa idosa, nota-se despreparo desses profissionais para o cuidado a essa população, configurando um desafio diante do contexto atual ${ }^{(11-12)}$.

O fortalecimento do ensino da enfermagem gerontológica é urgente, assim, acredita-se que a criação do Departamento Científico de Enfermagem Gerontológica da Associação Brasileira de Enfermagem consiste em uma mola propulsora. Esse departamento constitui-se como importante representante da área, para o debate de políticas de formação no contexto da própria Enfermagem para o cuidado a pessoa idosa, assim como na discussão de políticas públicas de atenção a este grupo populacional (13)

Contudo, ainda existem lacunas como a não obrigatoriedade de disciplinas relacionadas à gerontologia nos cursos de nível técnico e graduação em enfermagem. Assim, a inserção da temática nos cursos encontra dificuldades pela escassez de conhecimento gerontogeriátrico, a ausência de sintonia da maioria das instituições de ensino com o processo de transição demográfica, além da inexperiência do corpo docente na área. Portanto, a frágil introdução dos conteúdos de gerontologia nos currículos faz com que os profissionais recém-egressos não disponham das competências mínimas para atendimento ao idoso ${ }^{(11)}$

\section{A pessoa idosa e as doenças infectocontagiosas}

Embora a pandemia da COVID-19 se constitua ainda objeto de estudo e investigação por pesquisadores do mundo inteiro, já é consenso entre a comunidade científica que a população idosa compõe o grupo de risco mais vulnerável e com maiores chances de infecção e mortalidade pelo novo coronavírus ${ }^{(14)}$

As causas relacionadas à maior exposição desse grupo populacional à contaminação pelo coronavírus e evolução da doença a um estágio crítico, se relacionam aos aspectos do processo saúde/doença da pessoa idosa, de acordo com o que a literatura científica tem divulgado até o momento sobre a COVID-19. Os processos de senescência e seus impactos gerados no sistema imunológico, a presença de comorbidades crônicas pré-existentes com destaque para as doenças cardiovasculares, respiratórias e metabólicas como o diabetes, vem sendo apontadas como fatores cruciais ao determinar o contexto de vulnerabilidade ao novo coronavírus ${ }^{(15)}$

O atual contexto pandêmico também suscita um alerta para a necessidade de políticas e programas de saúde relacionados às doenças infectocontagiosas, uma vez que, apesar da transição epidemiológica brasileira apontar um aumento na prevalência de doenças crônicas, as doenças infecciosas ainda apresentam números elevados na população e sempre estiveram presentes no contexto de vida da pessoa idosa configurando-se como uma das principais causas de hospitalizações de idosos no Brasil(16)

Sabendo-se que os pacientes idosos possuem demandas específicas da sua fase de vida, questiona-se: os profissionais de enfermagem nas unidades hospitalares voltadas para tratamento de doenças infectocontagiosas possuem abordagens distintas ao cuidar de pacientes idosos e dos mais jovens? Eles foram preparados para reconhecer essas peculiaridades das necessidades de cuidado dos mais velhos?

Esses questionamentos são importantes na medida em que o difícil manejo do tratamento específico para algumas doenças infecciosas em idosos, em razão de efeitos colaterais dos fármacos, toxicidade hepática e neurotoxicidade podem implicar em maior permanência no ambiente hospitalar e elevados custos para 
as instituições, configurando-se como um problema não só para o próprio idoso como também para os serviços de saúde ${ }^{(17)}$.

Falando especificamente da pandemia atual, as medidas de prevenção levantadas pela COVID-19 como estratégia para a erradicação do vírus - como a mudança de comportamentos individuais e coletivos priorizando o bem-estar e o equilíbrio das condições higiênicas e sanitárias de saúde - devem ser incentivadas e incorporadas às rotinas de cuidado da população geral e idosa, visando o combate às doenças infectocontagiosas ${ }^{(6)}$

A despeito das melhorias alcançadas nas últimas décadas em decorrência de um modelo de atenção voltado para a promoção da saúde e de um sistema de saúde universal, integral e equânime, não se pode deixar de lembrar que a população idosa brasileira ainda apresenta demandas não atendidas, especialmente devido à sua vulnerabilidade a condições infecciosas até então, associadas majoritariamente a população jovem, a exemplo das Infecções Sexualmente Transmissíveis (ISTs), onde merece destaque os índices de infecção pelo HIV causador da Síndrome da Imunodeficiência Adquirida (Aids) nessa população, cresce significativamente ${ }^{(18)}$

Ao tratar das ISTs, percebe-se ainda, a resistência do idoso em falar abertamente sobre aspectos da sexualidade, movidos por conceitos cercados de tabus e estigmas construídos socialmente e culturalmente ao longo da vida ${ }^{(19)}$. Essa resistência se faz presente ainda entre os profissionais de saúde, pois, além do idoso não se mostrar confortável para falar de temas como este, encontram outras barreiras, como a própria deficiência de conhecimento e dificuldade na abordagem dos profissionais. Esse déficit se inicia em algumas realidades ainda no processo de formação dos profissionais de saúde, ao perceber temáticas que envolvem a sexualidade como um requisito optativo ou ainda que não esteja incluso nas estruturas curriculares ${ }^{(20)}$.

Nesse sentido, há uma urgência em sensibilizar e gerar uma aproximação durante a formação do profissional de enfermagem acerca dos aspectos relacionados à sexualidade, o que pode favorecer a prevenção de doenças infecciosas como as ISTs. As consultas de enfermagem, por meio dos programas de atenção à saúde do idoso, como o HiperDia, as atividades de educação em saúde, constituem ferramentas importantes na atuação do enfermeiro e são espaços favoráveis às ações de promoção do cuidado e prevenção de agravos na Atenção Primária à Saúde(21).

Ao pensar nos fatores de risco, em muitas realidades ainda se vê a ausência de conhecimento relacionado à adoção de comportamentos preventivos individuais e coletivos voltados às doenças infectocontagiosas, especialmente para esse grupo populacional ${ }^{(13)}$

Apesar da DCN não falar diretamente das doenças infectocontagiosas na saúde da pessoa idosa, esta apresenta pressupostos ligados a esse cuidado, quando afirma que o enfermeiro deve ser capaz de conhecer e intervir sobre os processos de saúde-doença mais prevalentes no perfil epidemiológico brasileiro e também ser capaz de conhecer as diferentes necessidades dos indivíduos nas diferentes fases da vida.

Desse modo, considera-se fundamental que a formação dos profissionais de enfermagem envolva a discussão do processo de transição epidemiológica no Brasil, refletindo sobre como algumas doenças infectocontagiosas foram erradicadas ou reduzidas, enquanto outras se mostram persistentes, emergentes e reemergentes na população ${ }^{(22)}$. Além disso, os conceitos de surto, epidemia e pandemia, e a forma como as equipes de saúde podem atuar em tais situações, também fazem parte do conhecimento necessário para enfrentamento das doenças infectocontagiosas.

Assim, a promoção do envelhecimento ativo, saudável, independente e autônomo deverá continuar sendo a prioridade das políticas voltadas a esse público, no entanto, o cenário pandêmico vivido na atualidade deixará como marcas a necessidade de atenção às doenças infectocontagiosas e suas manifestações na população idosa(23).

\section{Perspectivas para o ensino de enfermagem gerontológica pós-pandemia}

Diante o contexto da pandemia de COVID-19, ressaltado pela vivência assistencial e docente, ancorado em reflexões sobre o processo de formação do enfermeiro generalista e técnico em enfermagem, identificaram-se 
na literatura científica, aspectos que necessitam ser incorporados ou ampliados na formação dos profissionais de enfermagem para o cuidado gerontológico, os quais serão detalhados nas subseções a seguir.

\section{Vulnerabilidade da pessoa idosa frente doenças infectocontagiosas}

Entende-se ser importante e necessário a presença do conteúdo sobre das doenças infectocontagiosas na formação dos profissionais de enfermagem, uma vez que estas apresentam-se como causa de morbimortalidade entre às pessoas idosas. Em um estudo realizado para se verificar as causas de morte e outros processos patológicos em idosos autopsiados, evidenciou que as causas de morte mais frequentes foram as cardiovasculares $(43,7 \%)$ e as infecciosas (31\%). Percebe-se assim, que a frequência das causas infecciosas nos idosos foi alta, sendo observado uma sobreposição das duas categorias de doenças ${ }^{(24)}$

Soma-se a este cenário a alta prevalência de Internações por causas sensíveis à Atenção Primária (ICSAP) em idosos, nas quais se evidencia a presença de doenças infecciosas que podem ser tratadas na Atenção Primária à Saúde evitando hospitalização do idoso e assim, reduzindo a exposição a riscos como iatrogenias e infecção relacionada à assistência a saúde: Entre os anos de 2014 e 2016, o número de ICSAP correspondeu a $30,90 \%$ de todas as internações de idosos e $16,36 \%$ dos gastos com internação no estado do Rio Grande do Norte. Entre essas, as maiores causas de ICSAP em idosos foram as pneumonias bacterianas $(19,92 \%)$ seguido de gastroenterites infecciosas e complicações $(17,52 \%)^{(25)}$.

A literatura aponta um risco sete vezes maior de pessoas idosas sofrerem internação por causas evitáveis, devido maior vulnerabilidade física e financeira, e pouca compreensão das práticas do cuidado preventivo ${ }^{(26)}$. Nesse sentido, tais achados reforçam a importância de incluir conteúdos referentes à prevenção e tratamento de doenças infectocontagiosas na pessoa idosa em todos os níveis de formação da enfermagem. Soma-se a esse panorama a especificidade do processo de envelhecimento em contextos específicos que maximizam a vulnerabilidade da pessoa idosa, como, por exemplo, a pessoa idosa em situação de rua e a pessoa idosa indígena.

\section{Pessoas idosas em situação de rua e envelhecimento indígena}

Pessoas idosas vivendo em situação de rua é uma realidade vista, principalmente, nos grandes centros urbanos, necessitando de estratégias de enfrentamento a essa situação que afeta inúmeros idosos. Envelhecer nas ruas é um processo excludente tanto pelas mudanças próprias do envelhecimento, como são acentuadas em grupos específicos como as mulheres idosas, pela vulnerabilidade de gênero, além das demais fragilidades como os rompimentos de vínculos familiares, sociais e de emprego, dentre outros ${ }^{(27)}$

Estar em situação de rua para a maioria dos idosos caracteriza-se em sofrimento e dor. Eles descrevem as vulnerabilidades a que estão expostos, principalmente em relação ao frio, à fome, à violência, à invisibilidade social e dificuldade de acesso aos serviços de saúde ${ }^{(19)}$ Como consequência, os danos provenientes das adversidades a que esta população está exposta são inúmeros, entre os quais incluem-se doenças infectocontagiosas associadas a condições que às potencializam como a desnutrição, abuso de drogas, exposição à violência, além da privação de necessidades básicas, entre tantas outras ${ }^{(28)}$

Por sua vez, o envelhecimento dos povos indígenas, também suscita atenção, uma vez que se trata de uma população específica, com cultura e valores próprios, e com maior vulnerabilidade social, em vista do percentual elevado de famílias que se enquadram na pobreza e na extrema pobreza Acerca das doenças infectocontagiosas, como, por exemplo, a COVID-19, levantamentos estatísticos apontam elevada vulnerabilidade dos povos indígenas. Esta condição é intensificada por fatores relacionados à carência de infraestrutura de saneamento; existência de domicílios com maior média de moradores; maior distância dos municípios com disponibilidade de leitos em unidades de terapia intensiva e respiradores; ausência nos Distritos Sanitários Especiais Indígenas (DSEls) e de meios de transporte rápido para remoção de casos graves ${ }^{(29-30)}$ 
Esse contexto torna-se preocupante pelos impactos que podem trazer ao modo de vida e cultura indígena, a qual apresenta a riqueza da vida em coletividades e da transmissão intergeracional de conhecimentos por meio das pessoas mais velhas ${ }^{(30)}$

\section{Idosos institucionalizados e as doenças infectocontagiosas}

Outro ponto que deve ser reforçado durante o processo de formação é a importância da adoção das medidas de biossegurança nas Instituições de Longa Permanência para Idosos (ILPIs). A presença de profissionais de enfermagem possibilita um cuidado mais qualificado e seguro ao idoso institucionalizado. Contudo, as ILPIs são ambientes de alto risco para doenças infectocontagiosas, como, por exemplo, COVID-19, devido à idade avançada e às condições de saúde crônicas frequentes dos residentes, bem como à movimentação de profissionais de saúde entre instituições ${ }^{(31)}$

A prevenção e controle de infecções devem ser implementadas pelos profissionais que atuam nestas instituições, com o objetivo de evitar ou reduzir ao máximo a transmissão de microrganismos durante qualquer assistência à saúde realizada ${ }^{(31)}$, minimizando assim os riscos à saúde dos idosos residentes e profissionais.

Por consequência, o uso correto dos Equipamentos de Proteção Individual (EPI) e seu descarte adequado, bem como o gerenciamento dos Resíduos de Serviços de Saúde caracterizam os princípios da biossegurança, atendendo às normas e exigências legais, desde o momento de sua geração até a sua destinação final. Os conteúdos relacionados à biossegurança estão previstos nas disposições legais de formação dos profissionais de enfermagem, mas sua abordagem incipiente traz como consequência prejuízos para uma prática profissional mais segura ${ }^{(32-33)}$

\section{Cuidado domiciliar à pessoa idosa}

As medidas de prevenção à contaminação comunitária impactaram a todos, porém influenciaram ainda mais a população idosa, no que se refere aos conflitos intergeracionais e mudanças nas relações familiares e nos hábitos de vida decorrentes da pandemia. Há de se destacar também o atual rearranjo domiciliar com famílias menores e multigeracionais ${ }^{(34)}$

Devido ao maior isolamento social necessário para prevenção da COVID-19, a assistência domiciliar precisou ser modificada, um serviço que costuma beneficiar, principalmente, idosos mais vulneráveis, o que indica a importância desse tipo de cuidado para o alcance da promoção da equidade na atenção à saúde no país e prestação de cuidados aos que mais necessitam ${ }^{(35)}$.

Na Estratégia Saúde da Família (ESF), o fortalecimento de vínculos entre usuário e profissionais é fundamental para que o cuidado ao idoso ocorra de forma ampliada, o que envolve tratamento medicamentoso, alimentação, a hidratação, a higiene e a integridade biopsicossocial. Esses aspectos precisam então ser amplamente abordados na formação do enfermeiro.

Nesse nível de atenção, a família tem importante papel e caracteriza-se como cuidadora informal, cujas ações influenciam diretamente na qualidade do cuidado prestado ao idoso. Este é um contexto de cuidado desafiador e que precisa ser sistematizado pelo enfermeiro e orientado pela equipe de enfermagem ${ }^{(36-37)}$.

Assim, o profissional em formação precisa ter contato com esse processo de gestão do cuidado, bem como com a educação em saúde, pois são ferramentas importantes no planejamento e realização das ações das equipes da ESF no cuidado a pessoa idosa e sua família, com potencial para gerar melhores desfechos clínicos em idosos ${ }^{(37)}$. No contexto específico das doenças infectocontagiosas, como a COVID-19, as ações de educação em saúde, por exemplo, devem envolver orientações sobre biossegurança, uso e descarte dos EPIs, cuidados com secreções, eliminações fisiológicas e higiene.

Por fim, vale refletirmos sobre o distanciamento e isolamento domiciliar. Este, quando necessário, precisa ser acompanhado na perspectiva de prevenção de complicações dos problemas de saúde existentes, controle 
de sinais e sintomas e efetivação do tratamento para a doença atual, e repercussões do próprio isolamento, como a depressão e comprometimento cognitivo.

\section{CONSIDERAÇÕES FINAIS}

Com a ocorrência da pandemia COVID-19, abrange-se a necessidade de maior ênfase na abordagem ao cuidado relacionado às doenças infectocontagiosas no ensino de enfermagem gerontológica. A imunosenescência e a presença de doenças crônicas tornam o idoso mais propenso às doenças infectocontagiosas, um quadro que se torna ainda mais preocupante quando se pensa em contextos específicos como do enveIhecimento indígena, da pessoa idosa em situação de rua, dos que se encontram institucionalizados como também dos domiciliados, esses últimos, pelas implicações do distanciamento imposto pela pandemia.

O contexto vivido atualmente tornou urgente a aquisição de novos conhecimentos para lidar com uma doença, até pouco tempo, desconhecida e que, conforme a literatura e as vivências profissionais têm mostrado, afeta os idosos de forma mais grave. Com isso, nota-se que a pandemia trouxe à tona que a abordagem de conteúdos referentes a doenças dessa natureza e suas manifestações na pessoa idosa parecem ser incipientes na formação dos profissionais de enfermagem.

Assim, a presente reflexão pretende trazer contribuições para o corpo de conhecimento da área, dando visibilidade de aspectos importantes para o fortalecimento do ensino de enfermagem gerontológica para a formação desses profissionais com vistas a promover a qualificação da assistência à pessoa idosa também na perspectiva da prevenção e cuidado em condições associadas às doenças infectocontagiosas.

\section{AGRADECIMENTO}

Ao Departamento Científico de Enfermagem Gerontológica da ABEn Nacional.

Ao Grupo de Estudos e Pesquisa em Enfermagem, Saúde e Envelhecimento da Universidade Federal do Rio Grande do Norte.

\section{REFERÊNCIAS}

1. Wang W, Tang J, Wei F. Updated understanding of the outbreak of 2019 novel coronavirus (2019-nCoV) in Wuhan, China. J Med Virol. 2020;92:441-447. https://doi.org/10.1002/jmv.25689

2. Ministério da Saúde (BR). Coronavírus - COVID-19 - O que você precisa saber [Internet]. Brasília: Ministério da Saúde; 2020 [cited 2020 Aug 01]. Available from: https://www.saude.gov.br/saude-de-a-z/coronavirus

3. Ministério da Saúde (BR). Secretaria de Vigilância em Saúde. Boletim Epidemiológico Especial. Doença pelo coronavírus COVID-19 [Internet]. Brasília: Ministério da Saúde; 2020[cited 2020 Aug 02]. Available from: https://saude.gov.br/images/ pdf/2020/July/30/Boletim-epidemiologico-COVID-24.pdf

4. Ministério da Saúde (BR). Secretaria de Vigilância em Saúde. Painel epidemiológico COVI-19 [Internet]. Brasília: Ministério da Saúde; 2020[cited 2020 Aug 02]. Available from: https://www.conasems.org.br/wp-content/uploads/2020/07/3.-cPAINEL-EPIDEMIOLOGICO-COVID_19.pdf

5. Veiga AMV. Imunidade e Envelhecimento. In: Freitas EV, Py L. Tratado de geriatria e gerontologia. Rio de Janeiro: Guanabara Koogan; 2017;2116-2127.

6. Hammerschmidt KSA, Santana RF. Health of the older adults in times of the covid-19 pandemic. Cogitare Enferm. 2020;25:e72849. https://doi.org/10.5380/ce.v25i0.72849

7. Polaro SHI, Montenegro LC. Fundamentals and practice of care in Gerontological Nursing. Rev Bras Enferm. 2017;70(4):6712. https://doi.org/10.1590/0034-7167.2017700401

8. Rodrigues RA, Bueno AA, Silva LM, Kusumota L, Almeida VC, Giacomini SB, et al. The teaching of gerontological nursing in Brazilian public higher education institutions. Acta Paul Enferm. 2018;31(3):313-20. https://doi. org/10.1590/1982-0194201800044 
9. Alves, JED. Envelhecimento populacional no Brasil e no mundo segundo as novas projeções da ONU [Internet]; 2020 [cited 2020 Aug 03]. Available from: https://www.portaldoenvelhecimento.com.br/author/joseeustaquiodiniz/

10. Ministério da Saúde (BR). Saúde da pessoa idosa: prevenção e promoção à saúde integral [Internet]. Brasília: Ministério da Saúde; 2020 [cited 2020 Aug 03]. Available from: https://saude.gov.br/saude-de-a-z/saude-da-pessoa-idosa.

11. Kletemberg DF, Padilha MI, Maliska IA, Villarinho MV, Costa R. The labor market in gerontological nursing in Brazil. Rev Bras Enferm. 2019;72(Suppl 2):97-103. https://doi.org/10.1590/0034-7167-2018-0178

12. Ministério da Educação (BR). Conselho Nacional de Educação. Resolução CNE/CES n.3, de 7 de novembro de 2001. Institui Diretrizes Curriculares Nacionais do curso de graduação em Enfermagem [Internet]. Diário Oficial da União. Brasília; 2001 [cited 2020 Aug 02] Available from: http://portal.mec.gov.br/cne/arquivos/pdf/CES03.pdf

13. Alvarez AM, Reiners AAO, Polaro SHI, Gonçalves LHT, Caldas CP, Unicovsky MAR. Scientific Department of Gerontological Nursing of the Brazilian Nursing Association. Rev Bras Enferm. 2013;66(esp):177-81. https://doi. org/10.1590/0034-7167.2016690326i

14. Leão LRB, Ferreira VHS, Faustino AM. The elderly and the pandemic of Covid-19: an analysis of articles published in newspapers. Braz J Develop. 2020;6(7):45123-42. https://doi.org/10.34117/bjdv6n7-218

15. Zhang W. Manual de Prevenção e Controle da Covid-19. São Paulo: PoloBooks; 2020.

16. Silva AR, Rattes TSR, Silva MFC, Mota ER, Santos EMA, Santos KOB, et al. Hospital care for the elderly: morbidity and mortality profile of the main causes of hospitalizations. Rev Saúde Coletiva [Internet]. 2019 [cited 2020 Aug 07];9:218-224. Available from: http://periodicos.uefs.br/index.php/saudecoletiva/article/view/4717/4231

17. Mesquita XBM, Freitas GPMR, Medeiros ACM, Freitas PA, Cardoso PMO, Campos RO. Hospitalization and complications in elderly patients admitted at infectious disease reference hospitals. Rev Epidemiol Control Infect. 2015;5(1):23-30. https:// doi.org/10.17058/reci.v5i1.5313

18. Ferreira CO, Davoglio RS, Vianna ASA, Silva AA, Rezende REA, Davoglio TR. Vulnerability to sexually transmissible infections in elderly users of a trial and counseling center. Arq Cienc Saúde UNIPAR. 2019;23(3):171-80. https://doi.org/10.25110/ arqsaude.v23i3.2019.6757

19. Uchôa YS, Costa DCA, Silva Jr APS, Silva STSE, Freitas WMTM, Soares CS. Sexuality through the eyes of the elderly. Rev Bras Geriatr Gerontol. 2016;19(6):939-49. https://doi.org/10.1590/1981-22562016019.150189

20. Reis IF, Sacramento NS, Saldanha RCO, Barbosa CLO, Guerra HS. Elderly and sexually transmitted infections: a challenge for prevention. Braz J Health Rev. 2020;3(2):1663-7. https://doi.org/10.34119/bjhrv3n2-028

21. Zanco MRCO, Melo SJF, Cardoso BC, Santos MCS, Silva MMS, Figueredo RC, et al. Sexuality of the elderly: main challenges for the nurse's role in primary health care. Braz J Health Rev. 2020;3(3):6779-96. https://doi.org/10.34119/bjhrv3n3-217

22. Teixeira MG, Costa MCN, Paixão ES, Carmo EH, Barreto FR, Penna GO. The achievements of the SUS in tackling the communicable diseases. Ciênc Saúde Coletiva. 2018;23(6):1819-28. https://doi.org/10.1590/1413-81232018236.08402018

23. Kalache A, Silva A, Giacomin KC, Lima KC, Ramos LR, Louvison M, et al. Aging and inequalities: social protection policies for older adults resulting from the Covid-19 pandemic in Brazil. Rev Bras Geriatr Gerontol. 2020;23(6):e200122. https://doi. org/10.1590/1981-22562020023.200122

24. Oliveira FA, Reis MA, Castro ECC, Cunha SFC, Teixeira VPA. Infeccious diseases as causes of death in autopsied elderly. Rev Soc Bras Med Trop. 2004;37(1):33-6. https://doi.org/10.1590/S0037-86822004000100009

25. Santos KMR, Oliveira LPBA, Barbosa IR. Hospitalizations due to primary care sensitive conditions in a population of older adults in the state of Rio Grande do Norte from 2008 to 2016. Rev Bras Geriatr Gerontol. 2019:22(4):e180204. http://doi. org/10.1590/1981-22562019022.180204

26. Dourado I, Oliveira VB, Aquino R, Bonolo P, Lima-Costa MF, Medina MG, et al. Trends in primary health care-sensitive conditions in Brazil: the role of the Family Health Program (Project ICSAPBrazil). Med Care [Internet]. 2011;49(6):577-84. http://doi.org/10.1097/mlr.0b013e31820fc39f

27. Mattos CMZ, Grossi PK, Kaefer CT, Terra NL. Ageing of elderly living in street situation in Porto Alegre City, RS, Brasil. Rev Kairós Gerontol. 2016;19(3):205-24. http://doi.org/10.23925/2176-901X.2016v19i3p205-224

28. Silva FP, Silva EC, Ferreira AL, Frazão IS. Patient safety: within the reach of the homeless? Rev Bras Enferm. 2020;73(5):e20190114. http://doi.org/10.1590/0034-7167-2019-0114

29. Quermes PAA, Carvalho JA. The impacts of assistance benefits on indigenous peoples: case study in Guarani settlements. Serv Soc Soc. 2013;116:769-91. http://doi.org/10.1590/S0101-66282013000400010 
30. Ponte AL, Alarcon DF, Kaingang JD, Santos RV. Vulnerabilidades, impactos e o enfrentamento ao Covid-19 no contexto dos povos indígenas: reflexões para a ação. Observatório Covid-19 Fiocruz [Internet]. 2020 [cited 2020 Aug 03]. https://portal. fiocruz.br/observatorio-covid-19

31. Menezes TMO, Delvalle R, Santos AMR, Siewert JS. Prevenção e controle de infecções por coronavírus em Instituições de Longa Permanência para idosos[Internet]. In: Santana RF. Enfermagem gerontológica no cuidado do idoso em tempos da COVID 19. ABEn; 2020;18-23 [cited 2020 Aug 07]. http://www.abennacional.org.br/site/e-book-geronto/

32. Rosa CS, Campos LS, Santana RF. Plano de gerenciamento de resíduos em Instituições de Longa Permanência (ILPI) para idosos durante a pandemia da COVID-19. In: Santana RF. Enfermagem gerontológica no cuidado do idoso em tempos da COVID 19. ABEn; [Internet]. 2020 [cited 2020 Aug 09];37-45. http://www.abennacional.org.br/site/e-book-geronto/

33. Carvalho AA, Cristina SM. The teaching of Biosafety in Nursing Undergraduation: a literature review. Rev Bras Enferm. 2007;60(5):569-72. http://doi.org/10.1590/S0034-71672007000500016

34. Garcia LAA, Santos GAS. The COVID-19 pandemic and the repercussions on health care of the Brazilian elderly. REFACS. 2020; 8(3):335-36. http://doi.org/10.18554/refacs.v8i3.4557

35. Wachs LS, Nunes BP, Soares MU, Facchini LA, Thumé E. Prevalence of home care and associated factors in the Brazilian elderly population. Cad Saúde Pública. 2016;32(3);e00048515. http://doi.org/10.1590/0102-311X00048515

36. Santos FB, Valente GSC. Systematization of nursing care and patient safety in the home environment. Enferm Foco. 2020;11(1):106-13. http://doi.org/10.21675/2357-707X.2020.v11.n1.2679

37. Rodrigues RAP, Bueno AA, Casemiro FG, Cunha AN, Carvalho LPN, Almeida VC, et al. Assumptions of good practices in home care for the elderly: a systematic review. Rev Bras Enferm. 2019;72(Suppl 2):302-10. http://doi. org/10.1590/0034-7167-2018-0445 